Pure Sciences

Oral

Abstract ID: 123

\title{
Universal reliance on LAL/TAL in bacterial endotoxin detection in drugs and parenteral - An ecological and economic perspective
}

\author{
Akbar John, B ${ }^{\mathrm{a}}$ | Jalal, K.C.A. ${ }^{\mathrm{b}} \mid$ Hassan, S. ${ }^{\mathrm{b}} \mid$ Kamaruzzaman, B.Y., ${ }^{\mathrm{b}} \mid$ Rozihan, M. ${ }^{\mathrm{c}}$ \\ aINOCEM Research Station (IRS), Kulliyyah of Science, International Islamic University Malaysia \\ ${ }^{b}$ Department of Marine Science, Kulliyyah of Science, International Islamic University Malaysia \\ 'Department of Aquaculture, Universiti Putra Malaysia (UPM), Malaysia
}

Introduction: Endotoxin detection and quantification in biological drugs and parenteral is a crucial step in helping us to be confident in process control (IPC) and product release decisions. Food and Drug Administration (FDA) approves either Limulus Amebocyte Lysate (LAL) /Tachypleus Amebocyte Lysate (TAL) derived from blue blood of horseshoe crabs to detect and quantify endotoxin. This in turn increases harvest pressure on wild crabs for biomedical bleeding practice which eventually leads to post bleeding mortality up to $30 \%$. In economic perspective, the LAL/TAL industry worth more than 50 million USD annually. Methods: Numbers of methods were adopted to enhance the accuracy of endotoxin detection, including gel clot, turbidimetric and chromogenic method. Results: However, all these methods require crabs either from wild or from captive for bleeding purpose. Though, various conservative measures are being taken, the habitat degradation and anthropogenic pressures on wild crab population is still beyond control and leads to unsustainable utilization of natural stock. Conclusions: Due to an increased demand of $\mathrm{LAL} / \mathrm{TAL}$, the present review paper addresses the issues related to Eco-economical viewpoint on sustainable utilization of wild stock for future LAL/TAL industry. It also addresses pros and cons of recent advanced sensor technology in rapid and low cost detection of endotoxin.

KEYWORDS: LAL, TAL, horseshoe crabs, endotoxin 